\title{
Molecular and Morphological Characterization of Wild and Cultivated Native Fragaria in Southern Chile
}

\author{
M. Gambardella, ${ }^{1}$ A. Cadavid, V. Díaz, and R.Pertuzé \\ Facultad de Ciencias Agronómicas, Universidad de Chile, Casilla 1004, Santiago, Chile
}

In southern Chile, $F$. chiloensis grows in wild and cultivated forms. The great diversity of germplasm has motivated researchers from all over the world to collect the Chilean strawberry in a search for useful traits for breeding purposes. According to Staudt (1962), the cultivated form of $F$. chiloensis spp. chiloensis f. chiloensis can be distinguished from other Fragaria taxa by its vigorous growth habit. Plants are normally dioecious with leaves that are thick, coriaceous and crenate. Flowers are up to $52 \mathrm{~mm}$ in diameter with six to seven (five to nine) somewhat undulated petals. Fruits are large, elongate-conic or more or less spherical. All stems have sparse, spreading hairs. Runners are very vigorous and up to $4 \mathrm{~mm}$ in diameter. The Chilean wild form, F. chiloensis spp chiloensis f. patagonica Staudt, is less vigorous with less glossy leaves and its peduncles and pedicels are covered with hair; its fruits are also smaller and cuneiform shaped.

While the overall characteristics of the two Chilean forma of strawberries have been described, considerable variation exists in the natural populations. For the last 7 years, it has been our goal to collect and describe the levels of diversity in those natural populations. The results could improve our understanding of the botanical classification of this species and uncover useful variability for breeding. This report describes a number of genotypes collected in several germplasm exploration trips.

\section{Materials and Methods}

During 1996, 1998 and 1999, prospecting trips were carried out in southern Chile, between latitudes $38^{\circ} 04^{\prime}$ (Contulmo) and $43^{\circ}$ 07' (Quellón), with the aim of collecting $F$. chiloensis germplasm (Fig. 1). A preliminary description of the plants in coastal, mountain habits, and in small orchards, has been described (Gambardella et al., 2000). The plants were evaluated for a number of morphological traits and their patterns of isozyme and RAPD variation using a cluster analysis (NTSYS-93)(Gambardella et al., 2000). Four enzyme systems were evaluated that were highly polymorphic and gave good resolution (PGI, LAP, PGM, EST). To determine the most appropriate primers to use in the RAPD analysis, a preselection was carried out using three samples of genomic DNA. According to the quality of the band, 17 primers were selected from a total of 80 .

\section{Results and Discussion}

The morphological patterns observed in the wild and cultivated populations are summarized in Table 1. Four major groups were observed according to their fruit color and origin: 1) cultivated white-fruited types, 2) cultivated red-fruited types, 3) wild red-fruited types from coastal habitats, and 4) wild redfruited types from mountain habitats.

Seven to eight zymograms were observed for each isozyme and these patterns corresponded to those found in $F$. ×ananassa cultivars. The dendrogram obtained when values of similarity were calculated did not show clearly differentiated groups, although the samples generally clustered together in the four major groupings due to their fruit color and origin (Table 1).
The dendrogram generated from the RAPD data (Fig. 2) was more clearly resolved than an isozyme one for the same group of plants (Gambardella et al., 2000). The selected primers generated a total of 102 polymorphic RAPD markers, which constitutes a significant level of polymorphism (Wilkie et al., 1993). Although one cannot clearly distinguish differentiated groups in the phenogram, gradual distances between them can be seen ( 0.7 to 1.0$)$, and an association can be observed with the groups shown in Table 1.

Results allowed us to establish four different types of $F$. chiloensis (Table 1). In the future, it will be necessary to carry out detailed studies with other techniques used by modern taxonomy to elucidate these aspects more accurately.

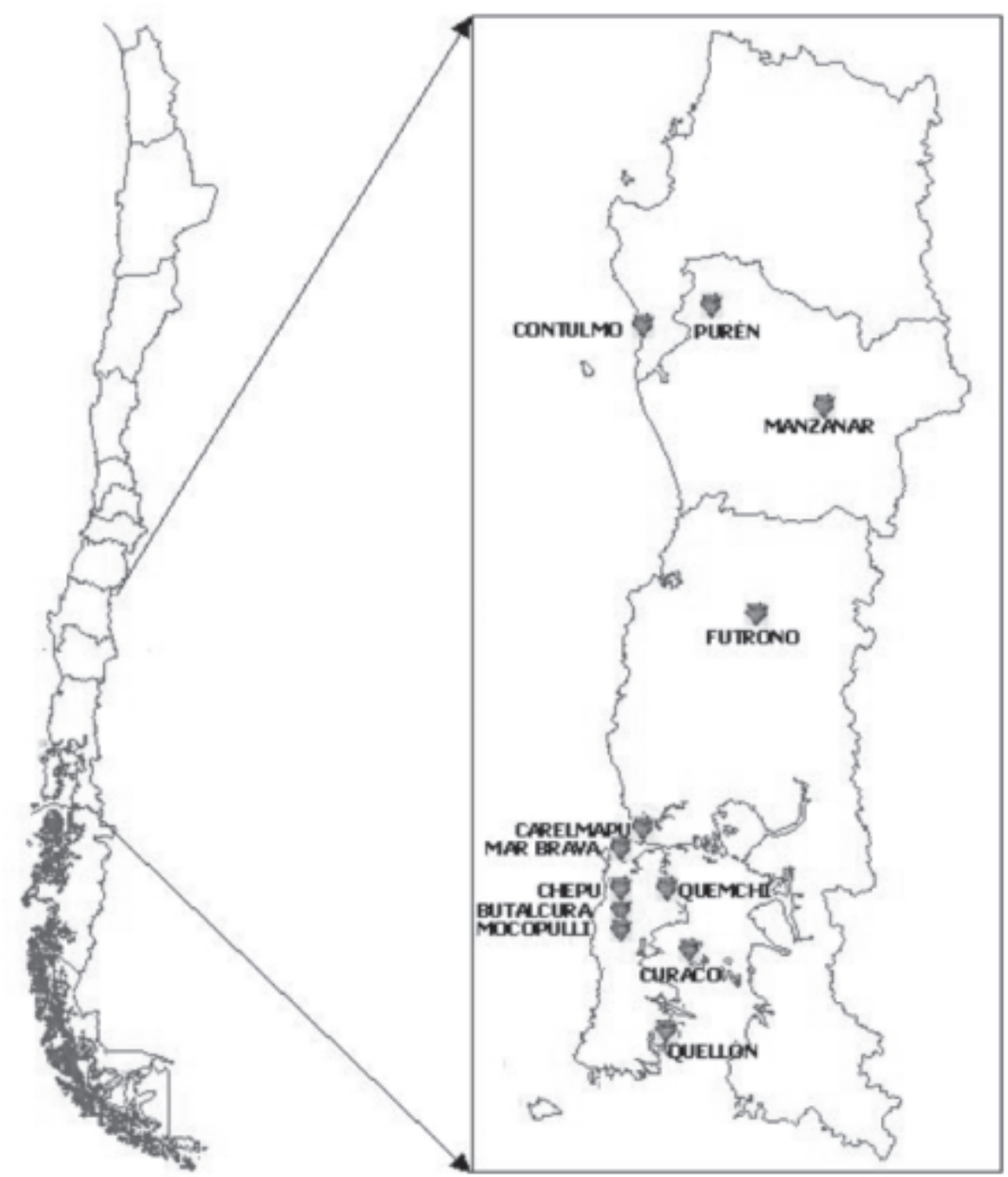

${ }^{1}$ To whom reprints should be addressed; e-mail mgambard@uchile.cl.

Fig. 1. Collection sites for Fragaria chiloensis in southern Chile. 
Table 1. Morphological types of Fragaria chiloensis according to characteristics and collection sites. Collections areas indicated on Fig. 1.

\begin{tabular}{|c|c|c|}
\hline Type & Collection sites & Morphological characteristics \\
\hline \multirow{2}{*}{ I. Cultivated white fruited plants } & & Petioles covered with lashed hairs \\
\hline & & Big fruits, white or pale pink \\
\hline \multirow[t]{3}{*}{ II. Cultivated red fruited plants } & Quemchi (Chiloé) & Medium vigor plants \\
\hline & Mocopulli (Chiloé) & Dark green and hairless leaves \\
\hline & Butalcura (Chiloé) & Small fruits, red or pink \\
\hline \multirow{3}{*}{ III. Wild plants, coastal habitat } & Chepu (Chiloé) & Dark green and hairless leaves \\
\hline & Curaco (Chiloé) & Small fruits, red \\
\hline & Carelmapu & Dioecious, monoecious and hermaphrodite \\
\hline \multirow{4}{*}{ IV. Wild plants, mountain habitat } & Futrono & Vigorous plants \\
\hline & & Leaves and petioles cavered with lashed hairs \\
\hline & & Medium fruits, red \\
\hline & & Dioecious, monoecious and hermaphrodite \\
\hline
\end{tabular}

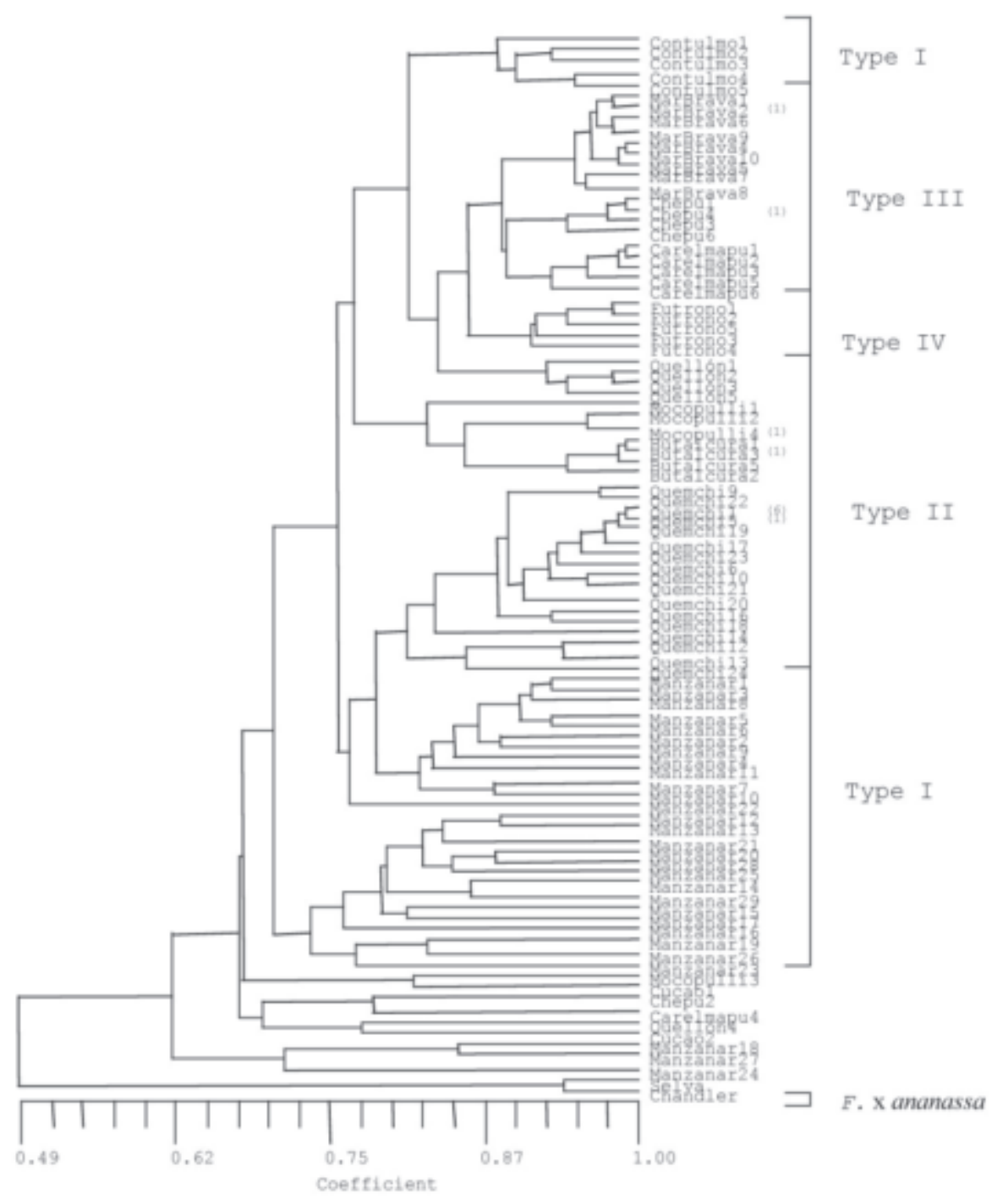

Literature Cited

Gambardella M, J.M. López-Aranda, W. Faedi, P.H. Roudeillac, and R. Infante 2000. Collection of wild and cultivated native Fragaria in southern Chile. Acta Hort. 567(1):61-63.

Gambardella M, A. Cadavid and V. Díaz. 2000. Isozyme and RAPD Characterization of wild and cultivated native Fragaria in southern Chile. Acta Hort. 567(1):81-83.

Staudt, G. 1962. Taxonomic studies in the genus Fragaria. Can. J. Bot. 40:869-886.

Wilkie S.E.,P.G. Isaac and R. J. Slater. 1993. Random amplified polymorphic DNA (RAPD) markers for genetics analysis in Allium. Theor. Appl. Genet. 86:497-5.

Fig. 2. Dendrogram of wild and cultivated strawberry accessions generated by cluster analysis of RAPD markers. 